\title{
LAS PALABRAS QUE TERMINAN EN EL SUFIJO -LANDIA Y SU TRADUCCIÓN AL ESLOVACO
}

\author{
Bohdan ULAŠIN \\ Universidad Comenius, Bratislava
}

\begin{abstract}
En): In this article, we analyse the formant-landia and the meanings it brings to the newly coined neologisms. As it is a relatively new element, there are still doubts about its classification since it exhibits both suffixal and compositional features. Different semantic uses are illustrated with real-life examples. One of the objectives of the article is also to search for parallel mechanisms in Slovak that are equivalent to the use of -landia in Spanish.
\end{abstract}

Keywords (En): -landia; suffix; coloquialism; word formation; neologism

Palabras clave (Es): -landia; sufijo; coloquialismo; formación de palabras; neologismo

DOI : $10.32725 /$ eer.2021.019

\section{Introducción}

El vocabulario y los mecanismos de su creación es el área donde se pueden observar de manera muy plástica muchas tendencias nuevas que aparecen sea por la necesidad de reflejar nuevos conceptos que van apareciendo constantemente en la realidad extralingüística, sea por los motivos de crear palabras nuevas, originales, no gastadas con las que nos referimos a entidades ya existentes. En este artículo queremos abordar la presencia del elemento -landia en las palabras creadas en español, de recién acuñación en casi todos los ejemplos encontrados, su uso y sus características denotativas y connotativas. Junto con el análisis vamos a buscar equivalencias funcionales en eslovaco para ofrecer una comparación de las dos lenguas mencionadas y ofrecer así un par de inspiraciones a la hora de buscar, caso de que surja la necesidad, posibles traducciones al eslovaco.

\section{Hipótesis}

A continuación formulamos dos hipótesis de partida sobre las características de las palabras creadas con el elemento-landia:

a) el formante-landia se va a añadir predominantemente a la base nominal (más del 95\%);

b) las palabras no serán de uso frecuente, estimamos que de más del $95 \%$ de ellas se documentarán menos de 10 casos en el corpus CORPES XXI y que ninguno de ellas presentará más de cien casos.

\section{2. ¿Sufijación o composición?}

Antes de todo, tenemos que abordar el problema de su clasificación morfológica, ya que el formante -landia podría formar parte de creaciones tanto sufijales como composicionales. En el DRAE se define como elemento

$$
-93-
$$


compositivo ${ }^{1}$. Seguro que es sobre todo por su origen léxico (véase más el capítulo 2 de este trabajo) que es un fuerte argumento para que se incluya dentro de la composición. Jesús Sánchez Lobato, a su vez, lo presenta como sufijo (SÁNCHEZ LOBATO, 2009: 189), también le asigna más rasgos propiamente sufijales Méndez Santos, pero prefiere clasificarlo como un sufijoide (MÉNDEZ SANTOS, 2012: 286287). Náñez lo designa como raíz sufija de términos que designan países (NÁÑEZ, 1973: 112) reflejando así la ambigüedad de su carácter derivacional-composicional. En la lingüística española hay varias inconsistencias acerca de la delimitación entre un sufijo y un elemento composicional, como bien lo analizó STEHLíK (2016, 4347).

El carácter sufijal, en base a la solución del DRAE con -oide ${ }^{2}$, aflora más en el significado despectivo que designa países o regiones con valor peyorativo y satírico: gringolandia, pijolandia, etc. y menos en el significado de 'tienda de'.

En este artículo trataremos el elemento -landia como sufijo, acorde con Sánchez Lobato, aunque somos conscientes de su posición límite entre la sufijación y composición.

\section{Origen y uso primario}

Por lo que se refiere al origen del sufijo -landia este viene de la palabra germánica: land $\left(<\right.$ protogermánico $\left.* l a n d j a^{3}\right)+-i a$ (sufijo toponímico románico y del latín, p. ej.: Valonia, Italia, Gal(l)ia, Hispania). El uso primario es, como ya se ha mencionado, designar países. No sorprende el hecho de que la mayoría son países o regiones de lenguas germánicas, en cualquiera de las dos variantes. El nombre original del topónimo ya contiene el elemento. Y se castellaniza al emplear una de dos variantes: -landia /-landa.

holandés: Holanda (Holland $<$ holandés antiguo holt landt 'tierra boscosa'4)

inglés: Nueva Zelanda ${ }^{5}(<$ holandés Nieuw Zeeland 'nueva tierra del mar', como referencia a la provincia neerlandesa Zelanda $<$ Zeeland)

lenguas nórdicas: Groenlandia (nórdico antiguo Grœnland 'país verde'), Islandia (islandés: Ísland 'tierra de hielo'), Jutlandia (danés Jylland, alemán Jütland 'país de los jutos'), etc.

O también lo encontramos en topónimos que llegaron al léxico castellano a través de una lengua germánica, la que añadió el -landia: Finlandia (sueco Finland 'país de los finlandeses', en finlandés Suomi), Tailandia (inglés Thailand 'país de los tailandeses' < tailandés thai + inglés -land), Suazilandia (inglés Swaziland 'país

\footnotetext{
${ }^{1}$ https://dle.rae.es/-landia [Consultado el 30. 11. 2019].

${ }^{2}$ Véase -oide que el DRAE define como elemento constitutivo con el significado 'parecido a', 'en forma de' (p. ej.: metaloide) y como sufijo en su acepción despectiva (p. ej.: feminoide) https://dle.rae.es/-oide [Consultado el 30. 11. 2019].

3 etymonline.com/search?q=land [Consultado el 30. 11. 2019].

${ }^{4} \mathrm{https} / / / \mathrm{www} . e t y m o n l i n e . c o m /$ search? $\mathrm{q}=$ holland [Consultado el 30. 11. 2019].

${ }^{5}$ Existe también la variante Nueva Zelandia. 
de los suazis' < suazi Eswati + inglés -land), Irlanda (< inglés Ireland < irlandés Éire $^{6}+$ sufijo inglés -land).

\section{1. Correspondencias en eslovaco:}

En caso de los topónimos no se trata de nombres originados en español, por lo tanto no se trata de equivalencias del formante español, lo que es el caso de los ejemplos del capítulo 5. No obstante, hemos decidido incluirlos aquí para ofrecer un panorama completo de su uso en español y sus correspondencias en eslovaco. Hay dos soluciones en eslovaco, la primera (más frecuente) consiste en reemplazar -land de la forma original por el sufijo eslovaco -sko que designa países. En la segunda se toma la estructura original con-land:

a) -sko: Ír-sko, Thaj-sko, Svazij-sko, Finn-sko, Holand-sko, Grón-sko, Jut-sko...

b) estructura original sin sufijos añadidos: Island, Nový Zéland...

\section{Aspectos formales:}

A veces los límites entre el núcleo y el sufijo son regulares: foto + -landia $=$ Fotolandia. En otros casos los límites necesitan una vocal de reajuste para enlazar los dos elementos, lo que va en contra de su clasificación como sufijo:

a) la vocal de enlace: -i-: Golpilandia, Tontilandia, Foxilandia (como en muchas composiciones más: blanquiazul, albiceleste, paticorto, cuellilargo, pelirrojo, piernilargo...);

b) a veces también figura como vocal de enlace la -o-: Chavezolandia, Tolkienolandia;

c) algunos ejemplos presentan más variantes: Jueguetelandia/Juguetilandia;

d) fusión de dos $l$ en los lindes en una $l$ : fútbol + -landia $=$ futbolandia;

e) acortamiento final del primer elemento: Mascota + landia = Mascolandia, jamón + landia $=$ jamolandia;

f) el acento normalmente cae en el sufijo -landia aunque no siempre, a veces también se documenta vacilación y existencia de dos variantes: télandia, futbolandia/ fútbolandia;

g) normalmente se escriben con mayúscula, siempre es así si la base es un nombre propio (Messilandia) o si el nombre de la tienda se considera como un nombre propio de la marca (Juguetelandia). Las creaciones aparecen en minúscula sobre todo si no se refieren a un país concreto y la base es un apelativo (aburrilandia).

\section{Otros usos:}

En el español actual el sufijo ha conocido una extensión notable, con dos usos principales:

1) la designación irónica, humorística de cierto país o región: Gringolandia, Tontilandia...;

\footnotetext{
${ }^{6}$ Del proto-céltico $* \bar{I} w e r j \bar{u}$, con el significado original de 'gordura', aquí en el sentido figurado de 'fertilidad' (MALLORY - AdAMS, 1997, 194). 
2) la designación de lugares de venta o de centros donde se ofrecen ciertos servicios o donde se desarrollan ciertas actividades: Zapatolandia, Jueguetelandia, Fotolandia, Librolandia...

En cuanto a la selección de las creaciones con -landia en español hemos tratado de hacer el inventario lo más extenso posible, incluimos aquí todas las creaciones del corpus de referencia CORPES XXI (El Corpus del Español del Siglo XXI ${ }^{7}$ ), el mayor corpus del español actual que abarca todos los países hispanohablantes. Hemos consultado su versión 0,92 que abarca más de 312 millones de formas que cubren los años 2001-2020.

Aparte del corpus arriba mencionado hemos decidido incluir también todas las creaciones que hemos sido capaces de encontrar durante nuestra investigación. En total son 153 creaciones de los que 88 se atestiguan en CORPES XXI y 65 no.

No incluimos aquí, claro está, los topónimos reales como Finlandia, Tailandia, Islandia, Groenlandia, u otros, menos conocidos: Cartolandia en México, Fordlandia, Uberlandia, Virgolandia en Brasil, etc.). Tampoco forman parte del inventario las palabras que se acuñaron en otras lenguas (p. ej. Disneylandia, parque de atracciones < inglés estadounidense Disneyland; Bowielandia, nombre de un libro < inglés Bowieland; el nombre de la película Lalalandia < inglés estadounidense Lalaland; Teletubilandia, país de los muñecos Teletubbies < inglés Teletubbyland; Thatcherlandia, el Reino Unido < inglés Thatcherland; Zombilandia $<$ inglés Zombieland; Alberolandia, parque de atracciones $<$ italiano albero 'árbol'; Bobolandia 'barrios bohemios y burgueses de París' < francés boboland, etc.), aunque si la base de estos anglicismos está traducida, sí que se incluyen en nuestro corpus (inglés Flatland $>$ Planilandia).

En caso de las 65 creaciones, las que no están en el Corpus, aparece la fuente documental que demuestra la existencia y la vigencia de la creación. En caso de las palabras que sí que quedan documentadas en el CORPES XXI añadimos la información acerca de la frecuencia, o sea el número de veces que aparecen en el corpus. La consulta del CORPES XXI no se incluye, es idéntica para todas las creaciones, el día 15 de diciembre de 2020.

\section{1. Topónimos:}

Este uso de valor claramente jocoso, muchas veces satírico y peyorativo (SÁNCHEZ LOBATO, 2009: 190) lo encontramos en palabras cuya creación se ve motivada por diferentes aspectos prototípicos o representativos del país / lugar en cuestión. Puede ser un topónimo concreto: Gringolandia 'Estados Unidos', o simplemente se refiere de manera genérica a cualquier sitio de dicha característica: aburrilandia 'sitio que aburre':

\footnotetext{
${ }^{7}$ REAL ACADEMIA ESPAÑOLA: Banco de datos (CORPES XXI) (en línea). Corpus del Español del Siglo XXI (CORPES XXI). http://www.rae.es
} 
a) según políticos o partidos políticos que lideran el país (casi siempre de connotación negativa e incluyendo el rechazo o la burla hacia la política que el personaje $^{8}$ o el partido en cuestión representa/representaba):

[1] Aznarolandia (España durante el gobierno de José María Aznar, 1996-2004)

"Recordamos Francolandia para impedir Aznarolandia".

Comentario en el foro: https://mx.answers.yahoo.com [Consultado el 30.11.2019]. ibérica)

[2] Borbonlandia (España, los Borbones son la dinastía real que encarna la monarquía

"La indignación colectiva no es un fenómeno exclusivo de Borbonlandia".

Blog (02.02.2014): http://blogdelviejotopo.blogspot.com/2014/02/la-sociedad-indignadafragmentos-en.html

[3] Bushlandia (Estados Unidos bajo la presidencia de George W. Bush, 2000-2008); CORPES XXI (1 caso)

[4] Chavezolandia (Venezuela, se refiere a la época de la presidencia de Hugo Chávez, 19992013 o a su legado en la actualidad)

"¿Seguro que todavía vivís en este país o ya os habéis trasladado a Chavezolandia?"

Comentario en el foro (16.03.2015): https:/www.meneame.net $/ \mathrm{m} /$ actualidad/c/16495934

[5] Felipelandia (la España del antiguo presidente de gobierno del PSOE, Felipe González, 1982-1996, o a partir del reinado de Felipe VI, 2014-)

"Un dirigente socialista ha criticado que quieran hacer de Catalunya una Pujolandia. Estoy de acuerdo. Pero me preocupa también la disyuntiva entre una Pujolandia estrecha y excluyente y un [sic!] Felipelandia".

Artículo periodístico: Francisco Umbral (20.09.1983):

https://elpais.com/diario/1983/09/20/sociedad/432856808_850215.html

[6] Foxilandia (México durante la presidencia de Vicente Fox, 2000-2006); CORPES XXI (4 casos)

[7] Francolandia (la España durante el franquismo o también la actual donde hasta hoy en día se nota el legado de la época de Francisco Franco, 1939-1975)

"Recordamos Francolandia para impedir Aznarolandia".

Comentario en el foro: https://mx.answers.yahoo.com [Consultado el 30.11.2019].

[8] Kirchnerolandia (Argentina, se refiere al gobierno de los presidentes Kirchner, Néstor y Cristina Fernández, 2003-2015)

"Malditos Kirchneristas, arruinaron Kirchnerolandia".

Artículo periodístico (11. 04. 2017) https://n.com.ar/politica/alicia-kirchner-nos-dejaronuna-provincia-quebrada_785430

[9] Pepelandia (España, de la sigla PP [pepe]: Partido Popular, se refiere al periodo entre los años 1996-2004 y 2012-2018 cuando el Partido Popular gobernó el país)

Los spots [sic!] publicitarios proclaman sin descanso en las pantallas de cada hogar pepelandés que el envidiable éxito obtenido por Pepelandia en la primera división de la Liga Globalizada de Naciones es un ejemplo para las democracias contemporáneas.

Artículo periodístico: Manuel Talens (09.04.2004):

https://elpais.com/diario/2004/03/09/cvalenciana/1078863484_850215.html

[10] Pujolandia (Cataluña, se refiere a esta comunidad autónoma a través del político Jordi Pujol i Soley, fue presidente de la Generalitat de Cataluña de 1980 a 2003)

"Un dirigente socialista ha criticado que quieran hacer de Catalunya una Pujolandia. Estoy de acuerdo. Pero me preocupa también la disyuntiva entre una Pujolandia estrecha y excluyente y un Felipelandia".

Artículo periodístico: Francisco Umbral (20.09.1983):

https://elpais.com/diario/1983/09/20/sociedad/432856808_850215.html

[11] Rajoylandia (España durante el gobierno de Mariano Rajoy, 2011-2018)

${ }^{8}$ No incluimos aquí los ejemplos que no surgieron en español sino en otras lenguas, como p. ej.: Putinolandia, Obamalandia, Trumplandia... 
Comenzamos la navegación. ¿Vienes? Próximo destino: Rajoylandia, antiguamente llamada España”.

Tuit (08.10.2014): https://twitter.com/hashtag/rajoynosmata

[12] Zapaterolandia (España durante el gobierno de José Luis Rodríguez Zapatero, 20042011)

"Zapaterolandia es el país en el que está mutando España gracias a la acción de gobierno de Zapatero. España, que, era un país con las características propias de una democracia europea, con Zapatero comienza a parecerse más a una república bananera. Esas repúblicas se caracterizan por el enorme divorcio que existe entre la realidad social y la realidad ciudadana".

Artículo periodístico: Román Cenodya (15.09.2008): https://www.elimparcial.es/noticia/23053/opinion/zapaterolandia.html

b) según otros personajes destacados (deportistas, escritores, actores...):

[13] Adelsonlandia (Las Vegas, según el nombre del magnate Sheldon Adelson, director ejecutivo de Las Vegas Sands Corporation); CORPES XXI (1 caso)

[14] Chapolandia (territorio donde reinaba el narcotraficante Joaquín Guzmán, conocido como El Chapo, líder del cartel Sinaloa, en la costa pacífica del norte de México); CORPES XXI (1 caso)

[15] Cristianolandia (el club del Real Madrid donde Cristiano Ronaldo, jugó un futbolista portugués, jugó como delantero entre los años 2009-2018)

"Isco brilla en Cristianolandia".

Artículo periodístico (23.09.2013): https://elmundano.wordpress.com/2013/09/23/iscobrilla-en-cristianolandia/

[16] Gabylandia (Argentina, según la famosísima tenista argentina Gabriela Sabatini, activa en los años 80 y 90); CORPES XXI (1 caso)

[17] Mahomalandia (cualquier país musulmán)

¿Y la diferencia entre Vasquilandia y Mahomalandia es ...?

https://plazamoyua.com/2015/01/08/y-la-diferencia-entre-vasquilandia-y-mahomalandia-es/ [Consultado el 30. 11.2019].

[18] Messilandia (el Futbol Club Barcelona, según el futbolista Lionel Messi que toda su carrera profesional ha desarrollado en este club, desde 2004)

¡Esto es Messilandia!

Portada del periódico Sport (16.02.2015)

[19] Mojamutilandia (país donde la gente se comporta como un personaje de animación Enjuto Mojamuto (creado por un actor español Joaquín Reyes), que está todo el tiempo delante de la pantalla conectado a Internet); CORPES XXI (1 caso)

[20] Spinettalandia (la palabra aparece en el álbum de un músico argentino Luis Alberto Spinetta Spinettalandia y sus amigos del año 1971); CORPES XXI (3 casos)

[21] Tolkienolandia (Nueva Zelanda, el país donde se rodó la trilogía El Señor de los Anillos basada en la obra de J. R. R. Tolkien, un filólogo y escritor británico)

"Poco a poco Nueva Zelanda empieza a convertirse en Nueva Tolkienlandia".

Blog (30.10.2012): https://formenelos.blogspot.com/?m=1

[22] Wagnerlandia (Bayeruth, según el compositor alemán Richard Wagner que vivió aquí de 1872 a 1882)

Nacimiento: Un pueblucho de Hungría. Defunción: En Wagnerlandia, Alemania

Entrada de una enciclopedia en línea https://inciclopedia.org/wiki/Franz Liszt [Consultado el 30. 11. 2019].

c) según los habitantes del país (normalmente se trata de algún rasgo (supuestamente) prototípico, a menudo negativo: panoli, pijo... o un sinónimo coloquial (también a menudo despectivo) de los habitantes del país: gringos, yanquis, gabachos, etc.: 
[23] Annunakilandia (se refiere a Estados Unidos, nombre de una canción de Calle 13, un grupo portorriqueño, se refiere metafóricamente a los annunakis, deidades sumerias y akkadias que esclavizan a los países que conquistan); CORPES XXI (1 caso)

[24] Cachacolandia (Bogotá, sobre todo en su acepción de la capital prestigiosa, de habitantes elegantes, ricos, a veces arrogantes hacia los demás; para los habitantes del caribe colombiano y venezolano, toda la parte interior del país, sobre todo las regiones andinas); CORPES XXI ( 1 caso)

[25] Cambalandia (región boliviana de la selva y de los llanos en el oriente, en los departamentos de Santa Cruz, Beni y Pando, viene de la palabra camba con la que se refería a la población indígena de los Kandire que habitaban la zona); CORPES XXI (1 caso)

[26] Cappylandia (en el doblaje de español latino el país de Cappies, los enemigos de Kirby, un personaje de dibujos animados japoneses); CORPES XXI (2 casos)

[27] Catrachilandia (sinónimo informal para Honduras, viene del gentilicio coloquial catracho, sinónimo de hondureño); CORPES XXI ( 2 casos)

[28] Chapinlandia (sinónimo informal para Guatemala, viene del gentilicio coloquial chapín, sinónimo de guatemalteco); CORPES XXI ( 3 casos)

[29] Charrolandia (nombre jocoso de la región de las tierras charras, del campo alrededor de Salamanca, el vocablo charro se refiere al aldeano salmantino); CORPES XXI (1 caso)

[30] Chicanolandia (el Suroeste de Estados Unidos que antes pertenecía a México y donde la cultura mexicana se siente hasta hoy en día. Los chicanos son los descendientes de mexicanos que viven en Estados Unidos, sobre todo en esta región fronteriza con México); CORPES XXI (1 caso)

[31] Chilangolandia (sinónimo argótico de la Ciudad de México, palabra de origen nahua); CORPES XXI (4 casos)

[32] Feolandia (sitio donde vive gente fea); CORPES XXI (1 caso) Unidos)

[33] Gabacholandia (en el español peninsular es Francia, en el español mexicano es Estados

Escucha y descarga gratis los episodios de Gabacholandia.. Nuestra corresponsal desde Francia nos manda su colaboracion

https://www.ivoox.com/podcast-gabacholandia_sq_f1266126_1.html [Consultado el 30.11.2019].

[34] Gallegolandia (en el español argentino se refiere a España, debido a la inmigración masiva de Galicia en el siglo XIX y XX, el gentilicio gallego empezó a usarse también como sinónimo de español); CORPES XXI (2 casos)

[35] Gitanolandia (España, especialmente la región andaluza donde viven muchos habitantes de procedencia gitana)

"Oye, fascista asqueroso, Gitanolandia nunca ha expedido pasaporte alguno".

Comentario en el foro (05.01.2019):

https://www.meneame.net/story/este-discurso-baltasar-pueblo-andoain-gipuzkoa-finalinesperado/standard $/ 2$

[36] Güerolandia (país de habitantes blancos, güero en el español mexicano significa 'persona rubia'); CORPES XXI (1 caso)

[37] Gringolandia (Estados Unidos, el país de los gringos); CORPES XXI (21 casos)

[38] Guirilandia (Gran Bretaña. Los guiris son extranjeros ricos, blancos que vienen a España como turistas)

Entradas sobre guirilandia escritas por barbarapadilla y bristoleno.

https://bristolenos.com/tag/guirilandia/ [Consultado el 30.11.2019].

[39] Gurulandia (país de los gurús)

Gurulandia, el país del Maestro Amor. Aunque fue condenado a 14 años de cárcel por abusos sexuales, el gurú Ricardo Ocampo seguirá ejerciendo su poder.

https://www.lavoz.com.ar/ciudadanos/gurulandia-el-pais-del-maestro-amor\#!/recuperar

[Consultado el 30.11.2019].

[40] Hadalandia (país ficticio de las hadas); CORPES XXI (1 caso)

[41] Latinolandia (mundo latino, de los latinos, de su cultura)

Que quiere decir "latinolandia"? No es un terreno sino la cultura hispana de Sudamerica y Centroamerica?

$$
-99-
$$


https://forum.wordreference.com/threads/latinolandia.794046/ [Consultado el 30.11.2019].

[42] Mariachilandia (México, el país donde uno de los símbolos es la música de los mariachis)

"Ethel Almada, la bailarina y actriz paraguaya que viene laburando desde hace meses en mariachilandia, se casó el jueves casi de sorpresa para muchos de sus familiares".

Artículo periodístico (08.09.2017): http://www.cronica.com.py/2017/09/08/actrizparaguaya-se-caso-mexico/

[43] Mañolandia (manera irónica y jocosa de referirase a Aragón, del gentilicio coloquial maño 'aragonés')

http://coloquialmente.com/es/manolandia [Consultado el 30.11.2019].

[44] Mariconlandia (país o lugar frecuentado por los homosexuales o un país donde tienen garantizados todos los derechos y esto se ve como algo negativo)

"Estoy aquí en casa de Antonio tan a gusto, me tiene hecho un príncipe, tanto es así que me encuentro mucho mas fuerte, no me canso casi y eso que vamos paseando a todos los lugares, por ejemplo, hemos estado en la tienda de Nieves allí viendo los libros y tomando un cafetito, esas cosas que me encantan como a buen marica de provincias que viene a la capital, a mariconlandia (chueca) [sic!] donde las musculosas y no tanto se pasean a sus anchas luciendo esos cuerpos que Dios y el gimnasio (y algún que otro anabolizante) les han dado".

Blog (agosto de 2010): http://absolutamenteyo.blogspot.com/2010/08/madrileando-ymariconeando.html

\section{[45] Morolandia (Marruecos)}

¿Que opinais de morolandia [sic!], digo Marruecos?

https://www.forocoches.com/foro/showthread.php?t=5615299 [Consultado el 30.11.2019].

[46] Paletolandia (país de los paletos, gente poco educada, de mente cerrada, rústica)

Los noticieros de paletolandia.

https://www.elimparcial.es/noticia/48438/opinion/los-noticieros-de-paletolandia.html

[Consultado el 30.11.2019].

[47] Panolilandia (país de los panolis, gente ingenua y fácil de engañar, aparece en un libro de Víctor Manuel Iglesias Retorno a Panolilandia)

$\mathrm{https} / /$ www.abebooks.com/RETORNO-PANOLILANDIA-Iglesias-Viqueira-Victor-

Manuel/22555594252/bd [Consultado el 30.11.2019].

[48] Pijolandia (país o lugar de los pijos)

"Urra habla del peso de la ley porque ha interiorizado lo que todos los articulistas de fondo: que Pozuelo es Pijolandia".

Artículo de prensa: Ignacio Ruiz Quintano (10.09.2009):

https://www.abc.es/opinion/abci-pijolandia-200909100300-1023994636179_noticia.html

[49] Pitufilandia (país de los pitufos, personajes de animación); CORPES XXI ( 2 casos)

[50] Prostitulandia (despectivamente referido a España); CORPES XXI (2 casos)

[51] Rapailandia (Brasil en el español paraguayo, rapai es del portugués rapaz 'chico'); CORPES XXI ( 2 casos)

[52] Princesalandia (sitio de web para niñas interesadas en princesas, muñecas)

http://princesalandia.blogspot.com/ [Consultado el 30. 11. 2019].

[53] Republicolandia (Estados Unidos según los ideales de los republicanos); CORPES XXI (1 caso)

[54] Spantilandia (país imaginario donde vive Spanton, un personaje de la obra Contarlo todo de un escritor peruano Jeremías Gamboa); CORPES XXI (1 caso)

[55] Tanolandia (sinónimo informal para Italia, en el español argentino se usa la palabra tano como sinónimo coloquial de italiano); CORPES XXI (2 casos)

[56] Tarzilandia (restaurante famoso en Caracas, Venezuela); CORPES XXI (19 casos)

[57] Ticolandia (sinónimo informal para Costa Rica, viene del gentilicio coloquial tico, sinónimo de costarricense); CORPES XXI (6 casos)

[58] Tontilandia (el país de los tontos)

"No es Tontilandia, es Cataluña. Infierno fiscal".

Título de un artículo periodístico (07.03.2016): https://lapaseata.net/2016/03/07/losdescartes-de-linda-galmor-de-hoy-pertenecen-al-peloteo-de-los-populistas-expertos-en-vendera-su-lider-mientras-tanto-en-barcelona/no-es-tontilandia-es-cataluna-infierno-fiscal/

$$
-100 \text { - }
$$


[59] Vasquilandia (País Vasco)

¿Y la diferencia entre Vasquilandia y Mahomalansia es ...?

https://plazamoyua.com/2015/01/08/y-la-diferencia-entre-vasquilandia-y-mahomalandia-es/ [Consultado el 30. 11.2019].

[60] Yanquilandia / Yankilandia (Estados Unidos, el país de los yanquis); CORPES XXI (15 casos)

[61] Yupilandia (país de los yuppies); (NGLE I, 2010: 786)

[62] Zorrilandia (nombre de varias páginas de porno, viene del significado coloquial de zorra 'puta'); CORPES XXI (1 caso)

[63] Zorrolandia (paisaje típico de la California mexicana, donde se desarrollan las aventuras del personaje ficticio El Zorro); CORPES XXI (1 caso)

d) según la actividad, fenómeno, objeto o animal o característica prototípicos del país:

[64] Aburrilandia (sitio que aburre); (MÉNDEZ SANTOS, 2011: 315)

[65] Bikinilandia (lugar o país de playas donde van a veranear muchos turistas)

"Famosas en Bikinilandia: analizamos a las 12 famosas con más bikinis en su maleta".

Portada de la revista Cuore (28.08.2018): https://www.facebook.com/cuore/photos/famosasen-bikinilandia-analizamos-a-las-12-famosas-con-más-bikinis-en-sumaleta/10155848306413543/

[66] Bocalandia (se refiere a la cavidad bucal de un cuento de La batalla de Bocalandia de un escritor uruguayo Alejandro Borges, en la que unos duendes pequeños protegen los dientes de los bacilos); CORPES XXI (1 caso)

[67] Cacalandia (país pobre, sucio, tercermundista, corrupto, donde no funciona nada); CORPES XXI ( 1 caso)

[68] Cuculandia (país imaginario de las fantasías sexuales, de cucu, cucuna 'vagina'); CORPES XXI ( 1 caso $)^{9}$

[69] Cangurolandia (Australia); CORPES XXI (2 casos)

[70] Caramelilandia (reino fantástico que aparece en el libro de Felipe Reyes Benítez El azar $y$ viceversa); CORPES XXI (1 caso)

[71] Ciberlandia (metafóricamente, sociedad cuyo funcionameniento depende de ordenadores y de Internet); CORPES XXI (1 caso)

[72] Colembolandia (costas antártidas donde viven muchos colémbolos, especie de artrópodos hexápodos); CORPES XXI (1 caso)

[73] Consumolandia (sociedad consumista)

Esta clase de sermoneo absurdo es típico en los escritores de Consumolandia.

http://www.tediumvitae.com/revista/no-1/manifiesto-del-lector [Consultado el 30. 11. 2019].

[74] Corruptolandia (país corrupto); (SANMARTíN SÁEZ, 2017)

[75] Crapulandia (lugar o país de crápulas, o sea borracheras y fiestas licenciosas); CORPES XXI (1 caso)

[76] Creditolandia (país en la que la gente tiene fácil acceso a préstamos gracias a sus tarjetas de crédito y se endeuda demasiado)

Zapatero el flautista de "creditolandia" o como no tener nunca culpa.

https://www.cronicaeconomica.com/zapatero-el-flautista-de-creditolandia-o-como-notener-nunca-culpa-6899.htm

[77] Cuatelandia (en Chile se designa así de manera coloquial y jocosa a México, de la palabra coloquial cuate que significa 'amigo' en México); CORPES XXI (1 caso)

[78] Culturilandia (lugares de cultura, como museos, pinacotecas, teatros, con cierto tono despectivo); CORPES XXI (1 caso)

[79] Cunillélandia (el mundo literario inventado por la dramaturga catalana Lluïsa Cunillé); CORPES XXI ( 7 casos)

\footnotetext{
${ }^{9}$ Aparece también en el libro de Carlos Fuentes: En esto creo. Barcelona, Seix Barral, 2002.
}

$$
\text { - } 101 \text { - }
$$


[80] dospuntocerolandia (sociedad o grupo de personas que siguen todas las novedades de la última generación del mundo de las nuevas tecnologías, el 2.0); CORPES XXI (1 caso)

[81] Espaciolandia (traducción española de Spaceland, un país inventado de la obra de Arthur Square: Planilandia: una novela de muchas dimensiones); CORPES XXI (1 caso)

[82] Espumalandia (país inventado de la obra del autor colombiano Juan David Correa Ulloa Todo pasa pronto, en el país, donde todo es muy blando, vivía el protagonista Cojín); CORPES XXI ( 5 casos)

[83] Futbolandia (país donde el fútbol tiene mucha importancvia sociocultural, en la mayoría de los casos se refiere a Brasil); CORPES XXI (3 casos)

[84] Goldeslandia (país inventado de la obra de un escritor boliviano Raúl Rivanadeira El retorno. El país es una alusión a Estados Unidos, la base tiene que ver con gold 'oro', como referencia a la vida consumista y materialista); CORPES XXI (4 casos)

[85] Golpilandia (un país donde se producen muchos golpes de estado, con más frecuencia se asocia tal vez con Bolivia)

"Bolivia, con justicia, se había ganado el calificativo de golpilandia y había sentado plaza de nación institucionalmente inestable".

Artículo periodístico (07.08.1985):

https://elpais.com/diario/1985/08/07/opinion/492213609 850215.html

[86] Impunilandia (país corrupto donde los políticos no respetan las leyes y se consideran impunes ante la justicia por más evidencia que haya de sus delitos, el caso recopilado del Corpus habla de México); CORPES XXI ( 1 caso) caso)

[87] Jaguarlandia (sinónimo coloquial de Chile en el español chileno); CORPES XXI (1

[88] Jamáslandia (úsase para ponderar la expresión jamás / nunca / jamás de los jamases (de jamáslandia)); CORPES XXI (1 caso)

[89] kurepilandia (Argentina en el español paraguayo, kurepi significa 'cerdo' en guaraní); (MÉNDEZ SANTOS, 2011: 317)

[90] Matalandia (cualquier sitio o lugar peligroso); CORPES XXI (1 caso)

[91] Narcolandia (Colombia, por el problema de narcotráfico que culminó en los años 80 y 90 y va unido sobre todo al nombre de Pablo Escobar); CORPES XXI (2 casos)

[92] Ningunalandia (país ficticio que no existe, juego de palabras que aparece en el libro del escritor mexicano César Huerta «Presentan una cinta sin música»); CORPES XXI (1 caso)

[93] Nuncalandia (país cuentístico imaginario presentado en el Festival infantil de Navidad); CORPES XXI (1 caso)

[94] Pactolandia (se refiere a España, también programa televisivo de La Sexta donde se analizó quién iba a gobernar después de las elecciones de 2019)

https://www.lasexta.com/programas/sexta-columna/quien-lograra-pactar-para-gobernarpactolandia-este-viernes-en-lasexta-columna-video_201905285cedb1a00cf22ed9c59cbae3.html

[95] Parlanchilandia (país o sitio donde la gente es muy habladora, parlanchina, en el español paraguayo se refiere a Paraguay); (MÉNDEZ SANTOS, 2011: 316)

[96] Pingüilandia (país de los pingüinos); CORPES XXI (1 caso)

[97] Planilandia (traducción española de Flatland, un país inventado de la obra de Arthur Square: Planilandia: una novela de muchas dimensiones); CORPES XXI (16 casos)

[98] Precariolandia (España, irónica y/o despectivamente, por ser país de trabajo y contratos precarios)

En esta tierra, transformada en Precariolandia a base de contratos parciales, reducciones salariales y convenios congelados cuando no a la baja, parece ser que el desparpajo ha calado en la casta dirigente y nos han regalado una peculiar interpretación de la realidad que no tiene desperdicio.

https://www.lahaine.org/est_espanol.php/31-chorradas-en-precariolandia [Consultado el 30. 11. 2019].

[99] Puntilandia (traducción española de Pointland, un país inventado de la obra de Arthur Square: Planilandia: una novela de muchas dimensiones); CORPES XXI (3 casos)

[100] Tranquilandia (una de las primeras acciones contra el narcotráfico en Colombia a principios de los 80 , el nombre se refiere al objetivo de lograr que Colombia fuese un país tranquilo); CORPES XXI (1 caso) 
[101] Trilandia (ciudad ficticia en un problema de la Olimpiada de Matemáticas de 2012 en Venezuela); CORPES XXI (3 casos)

[102] Tumulandia (país con muchos túmulos, palabra que en Centroamérica designa badén, un obstáculo en la carretera para que los vehículos se vean obligados a reducir la velocidad)

"Me voy a hospedar en Santa Cruz y bajare a Coban a turistear pero ahorita voy todavia por tumulandia".

Tuit (24.05.2013): https://twitter.com/ronald_mackay/status/338094849083469825

e) según el lugar:

[103] Españilandia (España, irónica y/o despectivamente)

Las cracias de Españilandia.

https:/www.periodistadigital.com/politica/opinion/columnistas/20160215/craciasespanilandia-noticia-689401433177/

[Consultado el 30. 11.2019].

[104] Eurolandia (la Unión Europea, despectivo y/o cómico); CORPES XXI (8 casos)

[105] Laboratoriolandia (país ficticio en un cuento de una alumna de $5^{\circ}$ de Educación Primaria del Colegio 'Rosalía de Castro', de Coslada (Madrid), Paola Castillo Guzmán, ha sido la ganadora del II Concurso de redacción '¿Qué son las vacunas para ti?’)

https://www.vacunas.org/ii-concurso-de-redaccion-sobre-vacunas-2/?print=print

[Consultado el 30. 11.2019].

\section{1. 1. Equivalencias eslovacas}

Para expresar semejante grado de innovación, satirismo e ironía usamos en eslovaco los siguientes procedimientos:

a) -stan (contiene la connotación de un país bananero, caótico, injusto, donde todo se somete a la voluntad del dictador), el origen de -stan es persa, con el significado de 'país', 'estado', lo encontramos en los nombres de varios países asiáticos: Kazajstán, Pakistán, Afganistán, etc. (LUTTERER - KROPÁČEK HUŇÁČEK, 1976: 206):

Banánistan (que tiene su motivación en la expresión banánová republika 'país bananero') Mečiaristan (según Vladimír Mečiar, el primer ministro eslovaco entre 1991-1998);

Slovakistan (Eslovaquia que se presenta así como un país subdesarrollado donde no funciona el estado de derecho y/o donde hay mucha corrupción);

b) -landia (país gobernado por): 2018);

Ficolandia (según Robert Fico, el primer ministro entre 2006-2010 y 2012-

Mečiarolandia (según Vladimír Mečiar, el primer ministro eslovaco entre 19911998);

c) diferentes juegos de palabras, deformaciones, cruce de palabras (predomina la intención lúdica y de originalidad):

Ficensko (un cruce léxico entre Fico + Slovensko 'Eslovaquia', se refiere a los años 2006-2010, 2012-2018 cuando Robert Fico fue el primer ministro eslovaco);

Pobrežie Slotoviny (la ciudad de Žilina, donde fue alcalde Ján Slota entre los años 1990-2006, cruce léxico de Slota + Pobrežie Slonoviny 'Costa de Marfil');

Slopensko (un cruce de las palabras Slovensko 'Eslovaquia' + slopat' 'emborracharse' que presenta a Eslovaquia como un país de borrachos); 
d): Král'ovstvo / Krajina $+\boldsymbol{N}_{\text {[G pl.] }}$ : Krajina hlupákov (krajina 'país', país de los tontos, Tontilandia), Královstvo lenivcov (královstvo 'reino', reino de los holgazanes);

e) otros sufijos (-ovo, -ovsko):

Zapadákovo (región, lugar subdesarrollado, un quinto pino);

Mečiarovsko (Eslovaquia gobernada por Vladimír Mečiar, el primer ministro eslovaco entre 1991-1998);

Romákovo (país de los gitanos, gitanolandia).

\subsection{Tiendas, empresas, asociaciones y proyectos}

El segundo uso del sufijo -landia significa una tienda especializada en vender un tipo de mercancía u ofrecer un servicio concreto. A veces el significado es más genérico que tienda, puede ser un lugar específico (plataforma en línea, etc.). Muchas veces el formante -landia añade el sema de 'tienda en la que existe una amplia oferta de cierta mercancía'. La motivación de estas palabras se basa casi exclusivamente en el primer caso. En la siguiente lista ofrecemos también el enlace a la página web de una tienda o lugar real con el nombre en cuestión:

a) según la mercancía vendida o actividades / servicios ofrecidos:

[106] Acualandia / Aqualandia (parque acuático); CORPES XXI (5 casos)

[107] Animalandia (parque de atracciones de animales); CORPES XXI (3 casos)

[108] Alfajorlandia (pastelería donde venden alfajores); CORPES XXI (1 caso)

[109] Biolandia (tienda de productos orgánicos y ecológicos)

https://es.linkedin.com/company/biolandia [Consultado el 30. 11. 2019].

[110] Burronlandia (parque temático en México, dedicado a la familia Burrón, historieta mexicana creada en 1948 por Gabriel Vargas); CORPES XXI (1 caso)

[111] Cappylandia (tienda de los zumos y bebidas de marca Cappy)

[112] Cevichelandia (restaurante donde sirven ceviche, plato a base de pescado o marisco crudo, muy popular en Ecuador o Perú); CORPES XXI (1 caso)

[113] Ciencilandia (proyecto telemático español destinado a los niños de 3 a 7 años, pretende poner en contacto a los niños con la ciencia); CORPES XXI (2 casos)

[114] Cinelandia (multicines); CORPES XXI (8 casos)

[115] Cuchilandia (restaurante en Perú, derivado de cuchara, cuchillo); CORPES XXI (2 casos)

[116] Cuerolandia (negocio de prendas y accesorios de cuero); CORPES XXI (1 caso)

[117] Discolandia (empresa discográfica boliviana); CORPES XXI (11 casos)

[118] Diverlandia (centro de ocio infantil); CORPES XXI (1 caso)

[119] Educalandia (una plataforma en línea para profesores)

educalandia.net [Consultado el 30.11.2019].

[120] Fantasilandia (parque de diversión en Chile); CORPES XXI (21 casos)

[121] Fiestilandia (salón de fiestas infantiles, empresa mexicana)

https://www.fiestilandia.com.mx/ [Consultado el 30. 11. 2019].

[122] Fotolandia (tienda dedicada a la fotografía en todo el proceso y a la venta de cámaras) https://www.facebook.com/pages/Fotolandia/1687183148200285 [Consultado el 30. 11. 2019].

[123] Gatolandia (organización sin ánimo de lucro, dedicada a la protección de gatos. De Zaragoza)

http://gatolandiazgz.blogspot.com/ [Consultado el 30. 11. 2019].

[124] Geolandia (nombre de proyecto educativo en Perú); CORPES XXI (1 caso)

[125] Jamolandia (restaurante que ofrece jamones y embutidos); CORPES XXI (1 caso) 
[126] Juguetelandia / Juguetilandia (juguetería, sala de juegos) https://www.juguetilandia.com/ [Consultado el 30. 11. 2019].

[127] Librolandia (librería, club de lectura)

http://premioespiral.org/recurso/librolandia/47f6c811-8834-4b76-95f5-ad87f70f2976

[Consultado el 30. 11.2019].

[128] Mascolandia (centro veterniario para mascotas)

https://mascolandia.com.co/ [Consultado el 30. 11. 2019].

[129] Navilandia (tienda colombiana que ofrece productos y artículos navideños); CORPES

XXI (1 caso)

[130] Orolandia (empresa de inversiones ${ }^{10}$ )

https://empresite.eleconomista.es/OROLANDIA-INVERSIONES-MADRID.html

[Consultado el 30. 11.2019].

[131] Poligolandia (proyecto didáctico de geometría para niños); CORPES XXI (5 casos)

[132] Pollolandia (restaurante de comida rápida especializado en comida de pollo); CORPES XXI ( 1 caso)

[133] Radiolandia (revista argentina que se publica bajo este nombre a partir del año 1934); CORPES XXI (26 casos)

[134] Saltilandia (centro de ocio para niños, con camas elásticas, castillos hinchables, etc.) https://www.facebook.com/SaltilandiaCoslada/ [Consultado el 30. 11. 2019].

[135] Sandwichilandia (lugar especializado en la venta de sándwiches)

http://ceipcaminodelnorte.centros.educa.jcyl.es/sitio/upload/PROGRAMA SEMANA CU

LTURAL_2018.pdf [Consultado el 30.11.2019].

[136] Teatrilandia (grupo venezolano de teatro); CORPES XXI (2 casos)

[137] Télandia (casa de té, tienda de té)

https://twitter.com/amoralte [Consultado el 30. 11. 2019].

[138] Titirlandia (asociación cultural de titiriteros, de España); CORPES XXI (4 casos)

[139] Tongolandia (tienda de calzado de marca mexicana Tongo); CORPES XXI (1 caso)

[140] Torolandia (parque infantil en España, donde los niños pueden, entre otras actividades, jugar al toro); CORPES XXI (2 casos)

[141] Zapatolandia (tienda de zapatos) 2019].

https://www.facebook.com/pages/Zapatolandia/956899231023771 [Consultado el 30. 11.

[142] Zumolandia (tienda / puesto donde venden zumos)

https://www.tripadvisor.sk/LocationPhotoDirectLink-g187472-d4334814-i132414408-

Zumolandia-Las_Palmas_de_Gran_Canaria_Gran_Canaria_Canary_Islands.html [Consultado el 30. 11.2019].

b) según el lugar donde se encuentra:

[143] Cortilandia (departamento infantil del Corte Inglés); CORPES XXI (1 caso)

[144] Esquilandia (parque de atracciones en Guatemala, del topónimo Esquipulas); CORPES XXI (10 casos)

c) según las personas a las que va dirigido:

[145] Chupilandia (centro de educación de niños pequeños); CORPES XXI (1 caso)

http://www.chupilandia.com/

[146] Frikilandia (tienda para frikis, de artículos frikis)

https://frikilandia36.webnode.es/ [Consultado el 30. 11. 2019].

[147] Emprendelandia (plataforma española de crowdfunding)

${ }^{10}$ En Italia es el nombre de joyerías. Por cierto, hay varias tiendas que encontramos tanto en Italia como en España (librolandia, cinelandia, etc.). Está fuera del objetivo de este artículo analizar si la lengua de origen es italiano o español. 
https://rincondelemprendedor.es/emprendelandia/ [Consultado el 30. 11. 2019].

[148] Infantilandia (librería y juguetería infantil española); CORPES XXI (1 caso)

[149] Pipilandia (empresa uruguaya dedicada al diseño y confección de prendas y accesorios para niños); CORPES XXI (1 caso)

[150] Princelandia (tienda de juguetes y ropa para niñas)

https://princelandiashop.com/ [Consultado el 30. 11. 2019].

[151] Profelandia (sitio web para maestros y profesores)

https://profelandia.com/ [Consultado el 30. 11. 2019].

d) otra motivación:

[152] Facilandia (tienda de electrodomésticos)

https://www.facilandia.com.py/ [Consultado el 30. 11. 2019].

[153] Macrolandia (macromercado mayorista con sede en Uruguay)

https://www.macromercado.com.uy/macrolandia/ [Consultado el 30. 11. 2019].

\section{2. 2. Equivalencias ${ }^{11}$ eslovacas}

a) Svet / Král'ovstvo / Raj $+N_{\text {[G pl.] }} ;$ Adj. + Svet / Král'ovstvo / Raj (se asocia con tiendas de amplia oferta de determinadas mercancías o servicios): Svet kníh (el mundo de los libros), Král'ovstvo hračiek (el reino de los juguetes), Svet farieb (el mundo de las pinturas / colores), Tortový svet (el mundo de las tartas), Raj kníh (el paraíso de los libros);

b) -landia: Tortolandia;

c) -land: Partyland (lugar donde se organizan fiestas de cumpleaños), Happyland (centro de ocio), Trikland (parque de atracciones de ilusiones ópticas);

d) con palabras inglesas (muchas veces tiendas de origen extranjero con sucursales en Eslovaquia): Jumping aréna (centro de ocio con camas elásticas).

\section{Cronología}

Este mecanismo de lexicogénesis es relativamente reciente, de las creaciones listadas en el inventario el primero es Cinelandia del 1938 (según el CNDHE, el Corpus del Nuevo Diccionario Histórico del Español ${ }^{12}$ ) y Radiolandia (título de una revista argentina) del año 1934 (CALZÓn FLORES, 1), luego hay unos cuantos casos de los años 50 (Tarzilandia) y empieza a crecer el número de creaciones de -landia en $\operatorname{los} 70$.

\section{Conclusión}

El sufijo -landia se ha establecido en el español actual extendiendo su significado original toponímico. Hoy en día se usa con el significado de 'tienda de', 'lugar en el que' y 'referencia humorística, a veces despectiva, de un país'. Es un procedimiento que se documenta también en italiano, portugués o inglés y a veces resulta difícil detectar la lengua de origen. Es preciso decir que el formante -landia

\footnotetext{
${ }^{11}$ Hablamos de equivalencias, no de traducciones porque en el caso de los nombres de las tiendas lo más correcto sería tratarlos como nombres propios y dejarlos sin traducir.

${ }^{12}$ Instituto de Investigación Rafael Lapesa de la Real Academia Española (2013): Corpus del Nuevo diccionario histórico (CNDH) (en línea). http://web.frl.es/CNDHE [Consultado el 15. 12 2020]. 
aporta carácter coloquial y original (a fin de captar la atención del cliente) al neologismo, en caso de las designaciones de países concretos (aparte de añadir el matiz coloquial) predomina el carácter irónico, burlesco, peyorativo, despectivo, hasta ofensivo de la forma-landia, lo que se puede observar en la elección de la base inicial expresiva (guiri, paleto, panoli, moro, gabacho, gringo, yanqui, etc.). En cuanto a la formación, siempre se pospone a la base, uniéndose a ésta bien directamente, bien a través de una vocal de enlace. En cuanto a su clasificación morfológica este formante presenta los rasgos tanto de proceso de sufijación, como de composición, si bien nos parece que el carácter sufijal es predominante.

Del análisis de los 153 vocablos con respecto a las hipótesis formuladas observamos que la mayoría de las creaciones se añaden a la base nominal (varias de ellas son bases adjetivonominales que pueden utilizarse de las dos maneras, p. ej.: Gallegolandia). En total 146 de 153, lo que equivale al 95,42\% del total. Los 7 restantes (el 4,58\%) son los siguientes: fácilandia (adjetivo fácil), jamáslandia (adverbio jamás), macrolandia (elemento compositivo culto macro-), ningunalandia (adjetivo indefinido ninguna), nuncalandia (adverbio nunca), precariolandia (adjetivo precario), trilandia (elemento compositivo culto tri-). En la primera hipótesis supusimos que las creaciones de la base nominal superaría el $95 \%$, la hipótesis se confirmó.

En la segunda hipótesis formulamos nuestra suposición acerca de la presencia de las creaciones de-landia en el Corpus CORPEX XXI. Pensábamos que más del 95\% de las creaciones no iban a aparecer en absoluto en dicho Corpus o iban a presentar menos de diez casos en él. Esta hipótesis no se confirmó, dado que 145 creaciones de 153 presentan menos de diez casos documentados de su uso en el Corpus CORPEX XXI, lo que equivale al 94,77\% de 1 número total:

65 creaciones de 153 no aparecen en el Corpus (el 42,48\% del número total de creaciones recopiladas):

50 de 153 aparecen $1 \mathrm{vez}(32,68 \%)$

13 de 153 aparecen 2 veces $(8,50 \%)$

6 de 153 aparecen 3 veces $(3,92 \%)$

4 de 153 aparecen 4 veces $(2,61 \%)$

3 de 153 aparecen 5 veces $(1,96 \%)$

1 de 153 aparece 6 veces $(0,65 \%)$

1 de 153 aparece 7 veces $(0,65 \%)$

2 de 153 aparecen 8 veces $(1,31 \%)$

De las 8 creaciones que aparecen 10 o más veces en el Corpus, he aquí su lista y el número de casos documentados: Esquilandia (10), Discolandia (11), Yanquilandia / Yankilandia (15), Planilandia (16), Tarzilandia (19), Fantasilandia, Gringolandia (21), Radiolandia (26). Así que ninguna de las creaciones ha superado los 100 casos.

Por lo que se refiere al eslovaco podemos decir que dispone de una amplia gama de mecanismos con los que se pueden conseguir las mismas intenciones lúdicas y rasgos coloquiales. 


\section{BIBLIOGRAFÍA}

CAlZÓN Flores, Florencia, Radiolandia en los cuarenta y cincuenta: una propuesta de entretenimiento (en línea)

http://www.unsam.edu.ar/escuelas/politica/centro_historia_politica/material/semin ario\%20CEHP.pdf [Consultado 15.12.2020].

Corpus del Español del Siglo XXI (CORPES XXI) (en línea). www.rae.es [Consultado 15.12.2020].

Corpus del Nuevo diccionario histórico (CNDH) (en línea) web.frl.es/CNDHE [Consultado 15.12.2020].

Diccionario de la lengua española (en línea) dle.rae.es, $23^{\mathrm{a}}$ edición [Consultado 30.11.2019].

KRÁLIK Libor (2015), Stručný etymologický slovník slovenčiny, Bratislava, Veda.

LUTTERER Ivan, KROPÁČEK Luboš, HuŇÁČEK Václav (1976), Původ zeměpisných jmen, Praha, Mladá Fronta.

MALlORY James P., ADAMS Douglas Q. (1997), Encyclopedia of Indo-European Culture, Londres, Fitzroy Dearborn Pub.

MÉndez SANTOS María del Carmen (2012), Los neologismos morfológicos del lenguaje periodístico, Vigo, Universidad de Vigo.

MÉNDEZ SANTOS María del Carmen (2012), Sobre -landia: origen, significado y comportamiento morfológico, in: Signo y Seña, 22, Buenos Aires, Facultad de Filosofía y Letras, p. 281-289.

NÁÑEZ, Emilio (1973), La lengua que hablamos. Creación y sistema, Santander, Bedia.

Online Etymology Dictionary (en línea) etymonline.com [Consultado 30.11.2019].

SÁncheZ LOBATO Jesús (2009), Procedimientos de creación léxica en el español actual, in: SÁnchez LobAto Jesús, Alba De Deigo Vidal, Pinilla GómeZ Raquel, Aspectos del español actual, Madrid, SGEL, p. 185-202

STEHLíK, Petr (2016), Problém delimitace některých slovotvorných postupi a prostředků ve španělštine, Brno, Filozofická fakulta Masarykova univerzita. 